\title{
The Electromagnetic Calorimeter of CMS
}

\author{
Wolfgang Funk on behalf of the CMS ECAL collaboration
}

\begin{abstract}
The electromagnetic calorimeter of CMS at the Large Hadron Collider (LHC) has been designed to measure the energy of electrons and photons with high resolution over a wide energy range. The detector consists of $61200 \mathrm{PBWO}_{4}$ crystals for the barrel and 14648 crystals for the endcaps, with a pseudo-rapidity coverage of $|\eta| \leq 3.0$. Preshower detectors are located in front of each endcap. The calorimeter has a pseudo-projective geometry. Great care has been taken in its design to minimize the effects of gaps and cracks in order to ensure the required energy resolution. The detector will operate in a magnetic field of 4 Tesla, and with a bunch crossing rate of $40 \mathrm{MHz}$. This requires fast and radiation hard crystals, photo-detectors and read-out electronics. An overview of the design, and the status of the construction, will be presented with special consideration given to the challenging goals.
\end{abstract}

Index Terms-Avalanche photodiodes, calorimetry, crystals, detectors, elementary particles, energy measurement, photodetectors, radiation detectors, scintillation detectors.

\section{INTRODUCTION}

$\mathbf{T}$ HE Compact Muon Solenoid (CMS) experiment [1] is one of two general purpose experiments being constructed for the Large Hadron Collider (LHC) at CERN. LHC will be a pp collider at a centre of mass energy of $14 \mathrm{TeV}$ and with a design luminosity of $10^{34} \mathrm{~cm}^{-2} \mathrm{~s}^{-1}$. Since the collider is running at a $40 \mathrm{MHz}$ frequency, we expect one crossing every $25 \mathrm{~ns}$. Each crossing of the beams, on average 20 events are produced, leading to about 1000 tracks at the same time in the detector. CMS has a magnetic solenoid field of $4 \mathrm{~T}$, in which the electromagnetic calorimetry [2] is placed. Our Level 1 trigger is designed to reduce the initial read-out rate of $40 \mathrm{MHz}$ to 10000 events per second. After software filtering, 100 events per second will be written to disk. The electromagnetic calorimeters have to survive a very harsh radiation environment of $3 \mathrm{kGy}$ and $10^{13}$ neutrons $/ \mathrm{cm}^{-2}$ for the barrel detector and up to $50 \mathrm{kGy}$ and $2 \times 10^{14}$ neutrons $/ \mathrm{cm}^{-2}$ for the endcaps.

\section{THE REQUIREMENTS For ECAL}

The main goal for the Electromagnetic CALorimeter (ECAL) is the precise energy and position measurements of electrons and photons. Assuming that the mass of the Higgs boson is below $150 \mathrm{GeV} / \mathrm{c}$, which is favored by precision electroweak measurements [3], the golden decay channel of the Higgs will be into 2 photons. Since the natural decay width of the Higgs is very small, the energy resolution of the calorimeter will dominate completely the observed width of the Higgs mass and therefore

Manuscript received November 4, 2004; revised September 1, 2006.

W. Funk is with CERN, CH-1211 Geneva 23, Switzerland (e-mail: wolfgang. funk@cern.ch).

Digital Object Identifier 10.1109/TNS.2006.880340

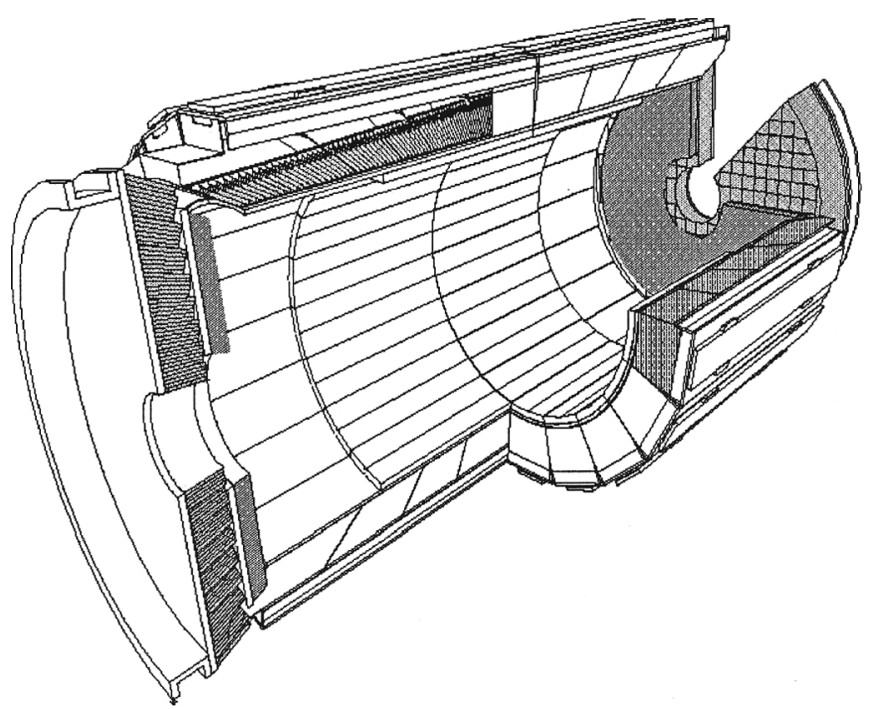

Fig. 1. Layout of the three ECAL sub-detectors with the $36 \mathrm{SMs}$ of the barrel, the 4 Dees of the endcap and the two disks of the preshower.

its discovery potential. In the case that the Higgs mass is above $150 \mathrm{GeV} / \mathrm{c}$ other decay channels such as $\mathrm{H} \rightarrow \mathrm{ZZ}$ or $\mathrm{H} \rightarrow \mathrm{WW}$, where the $\mathrm{Z}$ or the $\mathrm{W}$ are decaying into leptons and in particular into electrons, become more important. In order to achieve a good mass resolution of the Higgs for those decay channels, very good energy and position resolution for electrons is mandatory. The energy resolution of a calorimeter can be described with the following formula:

$$
\frac{\sigma(E)}{E}=\frac{a}{\sqrt{E}} \oplus \frac{b}{E} \oplus c
$$

where ' $\mathrm{a}$ ' is called the stochastic term, which includes the shower fluctuations, the photo-statistics and the fluctuations of the transverse leakage of the produced shower in the calorimeter. The design value of ' $a$ ' for our calorimeter was defined to be $2.7 \%$ and $5.7 \%$ for the barrel and the endcap calorimeter, respectively. The second term ' $\mathrm{b}$ ' is the noise term and comprises the electronics noise, the dark current and the pile up due to overlapping events. Using a $5 \times 5$ matrix for the cluster reconstruction we aim for $155 \mathrm{MeV}$ for the barrel and $770 \mathrm{MeV}$ for the endcaps at low luminosity. For high luminosity we expect $210 \mathrm{MeV}$ for the barrel and $915 \mathrm{MeV}$ for the endcaps. The third term ' $c$ ' is the constant term and describes the calibration precision, the longitudinal non-uniformities of the light collection, the fluctuations due to temperature and high voltage and the longitudinal leakage of the showers due to restricted length of the calorimeter medium. For both barrel and endcaps we try to achieve a constant term of $0.55 \%$. 


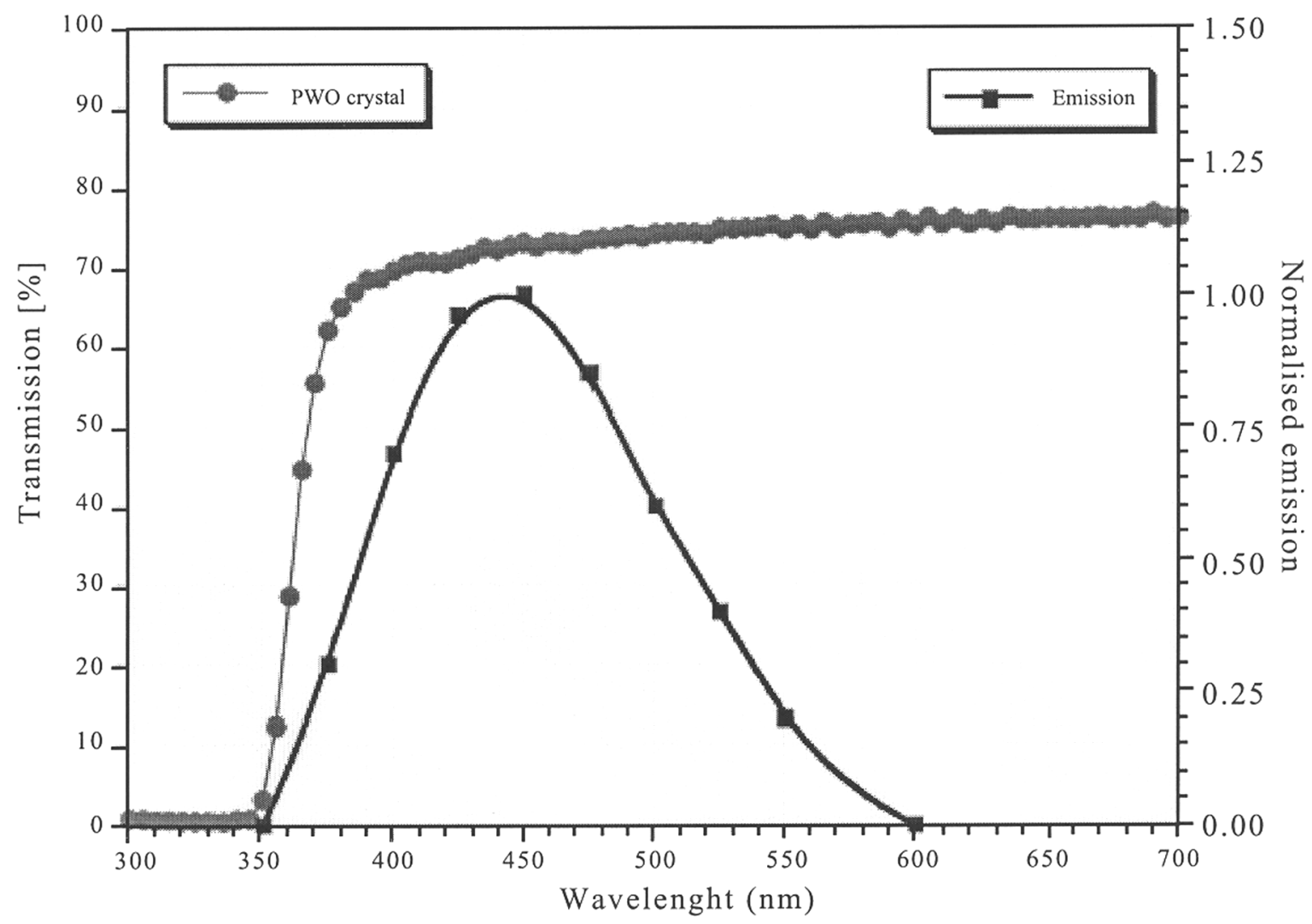

Fig. 2. Longitudinal transmission and emission spectrum versus wavelength for $\mathrm{PbWO}_{4}$ crystals of CMS.

\section{Choice OF THE CRYSTAl CAlorimeter}

We have chosen as calorimeter material lead tungstate $\left(\mathrm{PbWO}_{4}\right)$ crystals. Lead tungstate crystals are a homogeneous detection medium with a short radiation length $\mathrm{X}_{0}$ of $0.89 \mathrm{~cm}$ and a small Moliére radius of $2.19 \mathrm{~cm}$. This allows us to have a compact design and a fine granularity of the calorimeter. In addition, lead tungstate is a fast scintillator, where $80 \%$ of the emitted light is collected within $25 \mathrm{~ns}$. The peak emission wavelength is $420 \mathrm{~nm}$. Lead tungstate is an intrinsically radiation hard material, which is required by the harsh environment in which our detector is placed. Unfortunately lead tungstate has a relatively low light yield compared to other commonly used crystals. In particular, its light yield is only about $1.3 \%$ of the light yield of $\mathrm{NaI}$. We expect in CMS about 4 to 5 photo-electrons per $\mathrm{MeV}$. Lead tungstate has a strong dependence on the temperature of the crystals of $-2 \% /{ }^{\circ} \mathrm{C}$ at $18^{\circ} \mathrm{C}$, which demands a temperature stability of the crystals better than $\pm 0.05^{\circ} \mathrm{C}$. We achieve the required uniformity of the light yield along the crystals by de-polishing one face of the crystals.

\section{LAYOUT OF ECAL}

The barrel of ECAL consists of 61200 crystals, which are organized in two rings of 18 super-modules (SMs) each. Each ring defines a half-barrel. The two half-barrels cover a pseudo-rapidity of $|\eta|<1.48$. The size of the crystals is $\sim 22 \times 23 \times$ $230 \mathrm{~mm}^{3}$ which corresponds to $25.8 \mathrm{X}_{0}$. The two endcaps are comprised of 14648 crystals and are organized in 4 "Dees", covering $1.48<|\eta|<3$. The endcap crystals are a little bit larger than the barrel crystals $\left(30 \times 30 \times 220 \mathrm{~mm}^{3}\right)$.
There is one preshower disk [4] in front of each Ecal endcap, which allows $\pi^{0} / \gamma$ separation. The silicon detectors (4300 in total) are organized in 2 planes per disk, the first has a $\mathrm{Pb}$-layer of $\mathrm{X}_{0}=1.9$ as absorber in front, the second one of $\mathrm{X}_{0}=0.9$. The silicon is operated at $-10^{\circ} \mathrm{C}$. The preshower also provides accurate $\mathrm{x}-\mathrm{y}$ position reconstruction at a level of $300 \mu \mathrm{m}$ [2] for electrons and photons across the endcaps.

The three sub-detectors are all shown in Fig. 1.

\section{CRYSTAls}

Before using the crystals in the assembly of ECAL, all of them are controlled by an automatic system to check if they match the acceptance specification for geometry and optical properties as light transmission, light yield and uniformity [5]. 6 years of R\&D have lead to crystal growth conditions which, besides ensuring good optical properties, lead to crystals where the radiation damage affects only the light transmission. The longitudinal transmission curve as a function of wavelength, compared to the emission spectrum, is shown in Fig. 2. Samples of crystals are irradiated during mass production with low $(0.15 \mathrm{~Gy} / \mathrm{h})$ and high dose rate $(250 \mathrm{~Gy} / \mathrm{h})$, which covers the full range of radiation levels inside CMS. Up to today almost half of the barrel crystals have been delivered.

\section{CAlorimeter CONSTRUCTION}

The crystals are slightly tapered in order to achieve a pointing geometry. The crystal axes in the calorimeter are tilted by $3^{\circ}$ away from the interaction point to minimize gap and crack effects between crystals. 


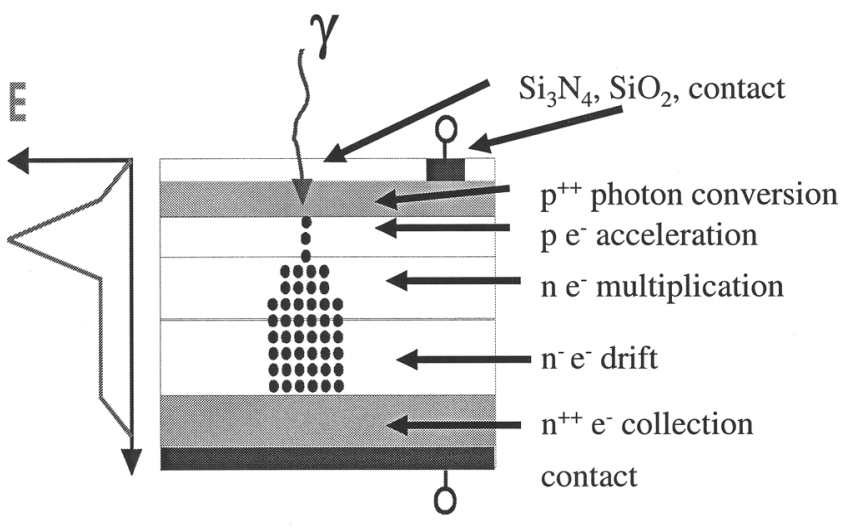

Fig. 3. Functional sketch of the APD.

For the barrel, $2 \times 5$ crystals are mounted in a fibreglass support structure, which has a low Z, to form a sub-module. 40 or 50, of those sub-modules are mounted together to form a module. To achieve the pointing geometry all crystals have their specific orientation. 4 modules are finally put together to form a super-module, which comprises $20 \times 85$ crystals. The weight of a super-module is about 3.5 tons. All the mechanical pieces and parts to mount the crystals inside the super-modules have been delivered. For the endcaps, super-crystals are formed with $5 \times 5$ crystals mounted in a carbon fiber structure. 156 of those are put on the back plate of each Dee with their specific orientation towards the interaction point.

\section{Photo-DeteCtors}

For the barrel, Avalanche Photo-Diodes (APDs) [6] as photodetectors are used, which were developed during an extensive R\&D together with Hamamatsu (S8141). They are solid state detectors, which are insensitive to magnetic fields. The structure of the APD is illustrated in Fig. 3. The photon, which was produced in the scintillation process, enters the APD and converts in the $\mathrm{p}^{++}$-layer. The electrons from the conversion are then accelerated in a gradient electrical field in the p-layer. The field peaks at the p-n junction and creates an avalanche multiplication. Afterwards, the produced electrons are drifting in a constant electrical field in a $\mathrm{n}^{-}$-layer towards a $\mathrm{n}^{++}$-layer, where they are collected as photoelectrical current. The active area of an APD is $5 \times 5 \mathrm{~mm}^{2}$. The quantum efficiency of $72 \%$ at $420 \mathrm{~nm}$ matches well the emission spectrum. Since the light yield of the crystals is low, a gain in the APDs of 50 is necessary. The required reversed bias voltage is $380 \mathrm{~V}$. Since the gain varies with-2.3\% $/{ }^{\circ} \mathrm{C}$ and $3.3 \% / \mathrm{V}$, a temperature stability of $\mathrm{T} \pm 0.05^{\circ} \mathrm{C}$ and a voltage stability of $\mathrm{V} \pm 20 \mathrm{mV}$ are required for the APDs. All APDs have been already produced and have passed through an extensive radiation test, where first the APDs were irradiated with a ${ }^{60} \mathrm{CO}$ source to $50 \mathrm{kGy}$. In the following annealing phase, the APDs were kept for 4 weeks at $80^{\circ}$ under bias voltage. Then, they were tested for their gain, dark current noise spectrum and break down voltage. $95 \%$ of all produced APDs passed this stringent test, which determines the most reliable devices. These devices will be inaccessible during the life of CMS. Two APDs are put into a capsule and then the capsule is glued on the rear face of each crystal.

The APDs develop high leakage currents after high levels of neutron irradiation and cannot be used in the region of $|\eta|>1.48$. Vacuum Photo-Triodes (VPTs) as photo-detectors are used for the endcaps [7], which are manufactured by RIE, St. Petersburg, Russia. They are single stage photo-multipliers and have an active area of $280 \mathrm{~mm}^{2}$. The quantum efficiency at $420 \mathrm{~nm}$ reaches $20 \%$. They need 2 voltages, $800 \mathrm{~V}$ and $1000 \mathrm{~V}$. They have a gain of 10 . The response in the magnetic field [8] has to be better than $85 \%$ at $4 \mathrm{~T}$ compared to no magnetic field. All VPTs are tested up to $1.8 \mathrm{~T}$ at an inclination to the B-field, which is representative of CMS. 10\% of all VPTs are tested to the full CMS magnetic field of $4 \mathrm{~T}$. They are radiation hard and their response decreases less than $10 \%$ after $20 \mathrm{kGy} .55 \%$ of all VPTs have been already received.

\section{READ-OUt ELECTRONICS}

The ECAL read-out electronics is organized in two layers. The first layer is the on-detector and the second layer is the off-detector electronics, which are connected together with an optical link system.

For the on-detector electronics, each trigger tower $(5 \times 5$ crystals) is served by 1 motherboard, 5 Very Front End (VFE) cards, one Low Voltage Regulator (LVR) card and 1 Front End (FE) card. The motherboard links together the APDs/VPTs with the high voltage supply and the 5 VFE cards, which preamplify, shape and digitise the signals with 3 different gains $(1,6,12)$ at $40 \mathrm{MHz}$ as shown in Fig. 4. A 12-bit ADC was developed by the ECAL collaboration leading to an effective dynamic range of about 15-16 bits. The tower energy is estimated on the FE card and sent to the trigger, whereas the data of the 25 channels is stored in the data buffer during this $\sim 3 \mu$ s of latency time. In parallel, the APD leakage currents and the temperature of every ten crystal are measured. All Integrated Circuits (ICs) are Application Specific Integrated Circuits (ASICs) in $0.25 \mu \mathrm{m}$ technology and are working within specification. All on-detector electronics for the barrel is in production.

The optical link system amounts to 13000 optical links at up to $800 \mathrm{Mbit} / \mathrm{s}$ at $1300 \mathrm{~nm}$ for the trigger, data and control information. The trigger and data optical links start at the FE card with the Giga-bit Optical Hybrid (GOH) chips and end about $100 \mathrm{~m}$ away at the off-detector electronics.

The off-detector electronics comprises Trigger Concentrator Cards (TCCs), which synchronize the trigger data and send it to the regional trigger system. The Selective Readout Processor (SRP) uses the trigger data from the TCCs to select the data for the normal read-out. The Data Concentrator Cards (DCCs) receive, reduce and format the data for the DAQ. The Clock and Control System boards (CCSs) provide clock, control and the trigger decision to the read-out electronics.

Very recently we installed the complete and final on-detector electronics including the optical link and cooling system inside the first final SM. After closure of the SM, we measured the noise of about 1000 channels at gain 12 and found on average $40 \mathrm{MeV}$ per channel, which is well inside our specification. 

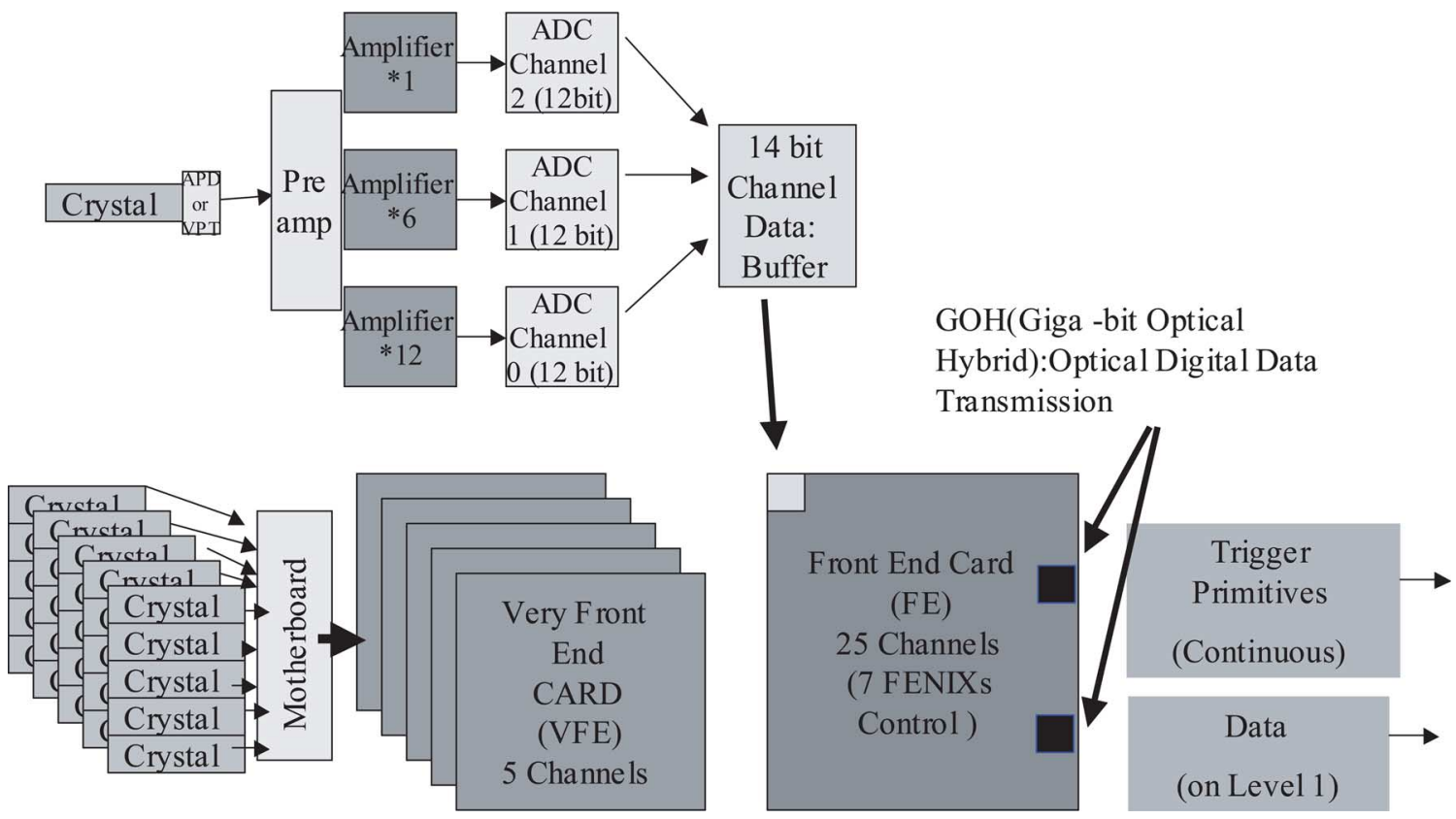

GOH(Giga -bit Optical

Hybrid):Optical Digital Data

Transmission

Fig. 4. Block diagram of the trigger tower electronics.

\section{Light MONITORING SYSTEM}

The light transmission of crystals changes under irradiation; the average effect is about $3 \%$ for a high luminosity run. Because of this we are obliged in CMS to monitor frequently the transmission with laser light of $440 \mathrm{~nm}$. In addition, we use laser light of $800 \mathrm{~nm}$ to monitor the stability of the electronics response [9]. The laser light is injected into the front (for the barrel) or into the rear (for the endcaps) part of the crystals by a laser monitoring system with a 2-level fanout architecture. The laser light will be injected during the $3 \mu$ s long abort gap of LHC, which will be present every $90 \mu \mathrm{s}$. The stability of the laser system itself is monitored with PN diodes to better than $0.1 \%$. It was demonstrated with the test-beam that the ratio of the change in response to the electrons in the beam to the laser light can be described by an universal constant $\alpha$ (see Fig. 5) [10]. During irradiation, in the plot, the response of all crystals is decreasing following the indicated universal line. During recovery the response increases on the same line. For all currently produced crystals, $\alpha$ was found to be 1.6 with an error less than 6 .

\section{Calibration Strategies}

Since the constant term ' $c$ ' is dominating the energy resolution at high energies and the constant term itself is mainly dominated by the inter-calibration precision between crystals, a large emphasis was put on the inter-calibration strategy in CMS.

For each crystal, the light yield is measured in the laboratory, using a ${ }^{22} \mathrm{Na}$ source. The test beam crystal calibration with an electron beam was found to be correlated with the measured laboratory light yields to a level of $4.6 \%$. Moreover, since the light yield and the longitudinal transmission at $360 \mathrm{~nm}$ are correlated, the combination of both measurements improves the inter-calibration precision to $4.1 \%$ [11]. The laboratory light yield data will be used to provide initial calibration constants at CMS for those elements, which were not calibrated at the test beam.

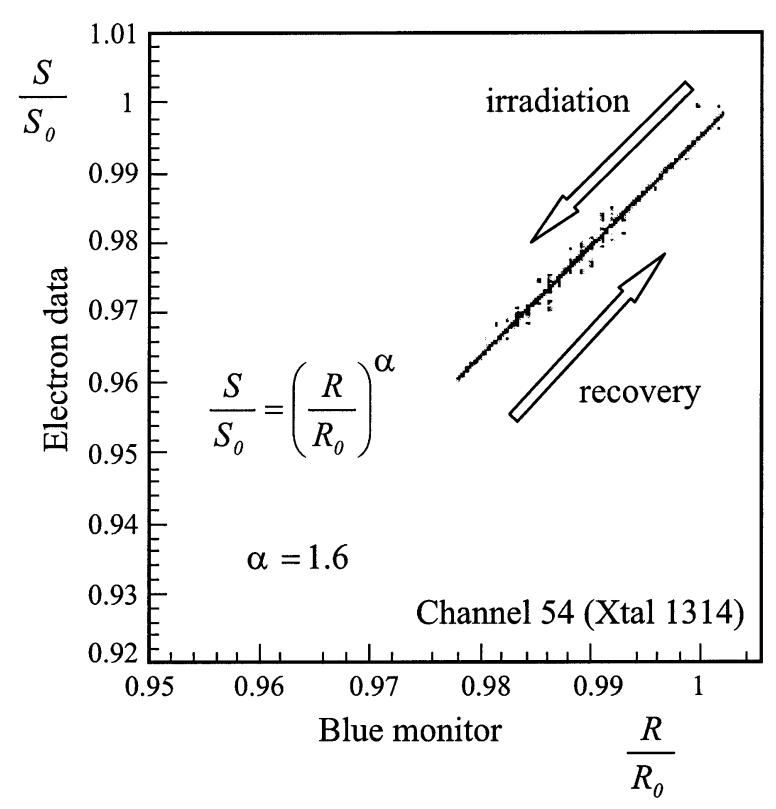

Fig. 5. The change in response of the electron signal versus the change in response of the laser signal, normalized with respect to their starting values, $\mathrm{S} / \mathrm{S}_{0}$ and $R / R_{0}$ respectively, due to irradiation. The ratio of the two is described by the universal constant $\alpha$.

A pre-calibration with electrons in the test-beam is expected to give 2\% accuracy in CMS. In October 2004, the first complete SM with all 1700 crystals will be calibrated in the test beam. Our goal is to measure as much as possible further SMs in 2006, if SPS is running in 2006. For the test-beam measurements electrons between 50 and $120 \mathrm{GeV}$ are required.

Recently, a new method for the inter-calibration, the so-called ' $\Phi$-symmetry calibration' [12] was studied for in-situ calibration in CMS. The idea is to inter-calibrate rings of equal $\eta$ with minimum bias events or with jet triggers, where the energy flow from the 2nd opposite jet is used to avoid trigger bias (see 


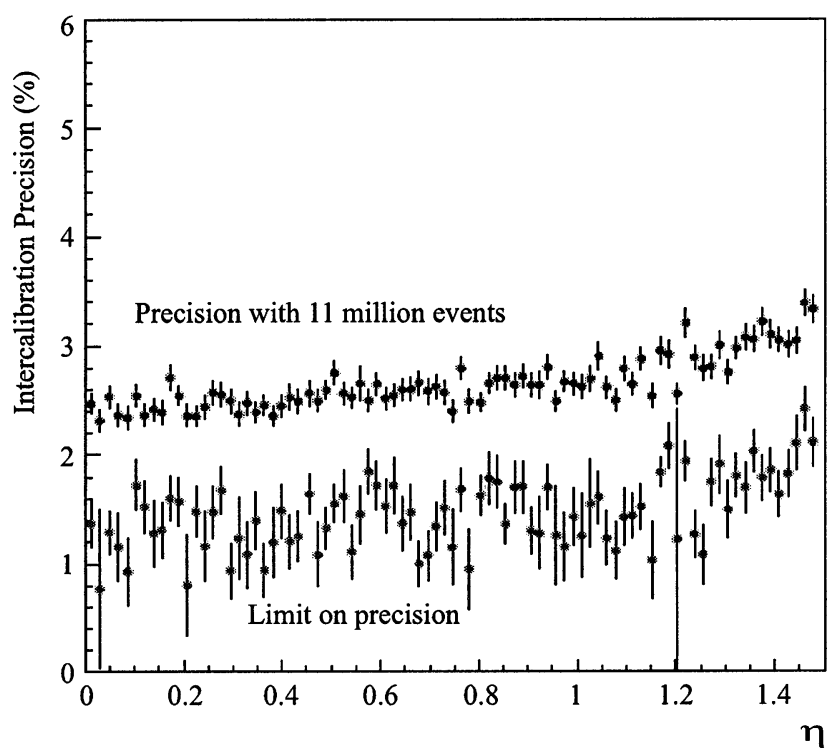

Fig. 6. Precision of the inter-calibration in \% versus the pseudo-rapidity with jet trigger events using the ' $\Phi$-symmetry calibration' method.

Fig. 6). The second step uses $\mathrm{Z} \rightarrow \mathrm{e}^{+} \mathrm{e}^{-}$events to inter-calibrate the rings of equal $\eta$. The same events are also used to determine the absolute calibration of the energy. This method was studied with great care with Monte-Carlo simulations and within a few days of stable running at LHC a precision of $2 \%$ should be achievable.

The ultimate calibration at LHC will be performed using E/p matching, using the ECAL and the Tracker with $\mathrm{W} \rightarrow \mathrm{e} \nu$ events. It has been demonstrated that this method depends only weakly on the quality of the starting calibration constants. At low luminosity $\left(\mathrm{L}=10^{33} \mathrm{~cm}^{-2} \mathrm{~s}^{-1}\right)$ an accuracy of $0.5 \%$ is expected within about 2 months of stable running conditions. For the momentum measurement, the Tracker of CMS has to be operational and aligned. The short-term changes of the calibration constants due to e.g., radiation effects will be determined via the laser of the monitoring system (Section IX).

\section{Energy and Position Resolution}

During the test-beam period in 2003, the energy resolution was measured with electrons using the final electronics for the barrel. In our test setup the electrons hit the centre of one crystal (a window of $4 \times 4 \mathrm{~mm}^{2}$ ). An energy resolution of $0.6 \%$ at $50 \mathrm{GeV}$ was reached by adding up the energy in a $3 \times 3$ crystal matrix (see Fig. 7) [13]. This result complies well with our design specifications. In the barrel detector position reconstruction is achieved by an algorithm based on energy weighting across neighbouring crystals. At the test beam the position resolution was determined to be up to $0.85 \mathrm{~mm}$ in $\mathrm{x}$ and $\mathrm{y}$ with $50 \mathrm{GeV}$ electron energy, which meets our specification. During the test beam activity starting in October 2004 the energy resolution of all crystals in a SM in the final layout will be measured.

\section{CONCLUSIONS AND OUTLOOK}

Electromagnetic calorimetry with high energy resolution is a central design feature of CMS. The $\mathrm{PBWO}_{4}$ crystals have production qualities within our specifications. The mechanics and

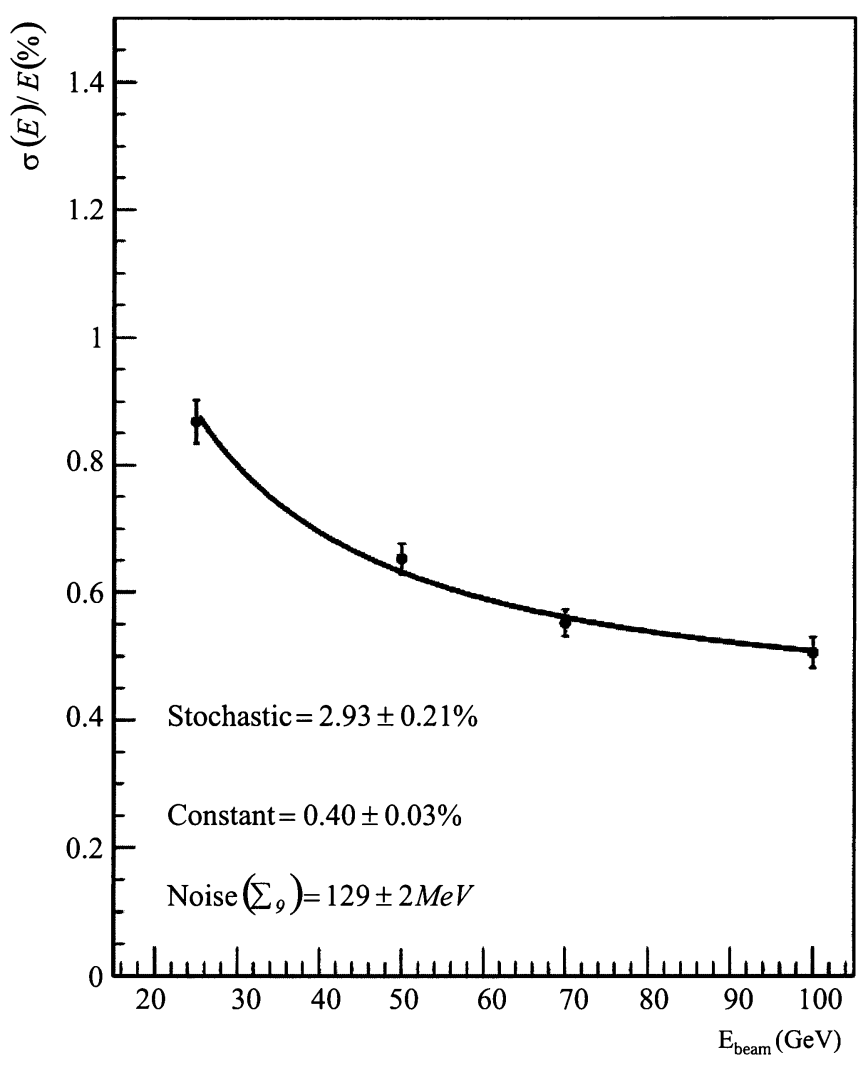

Fig. 7. Energy resolution measured with electrons applying a $4 \times 4 \mathrm{~mm}^{2}$ window in the centre of the crystal, which was hit by the electron, by adding up the energy in a $3 \times 3$ crystal matrix.

the photo-detectors are ready or under production. The mass production of the electronics is in progress, the integration of the electronics has just started with the first complete SM. The electronics has achieved its specified performance. The laser light monitoring system is working as expected.

Calibration strategies have been developed without requiring each SM/Dee to be in the electron test-beam. The test-beam results confirm the ambitious design goals of ECAL. In October 2004 the first complete SM will be operated in the test-beam.

\section{ACKNOWLEDGMENT}

It is a great pleasure for me to thank my colleagues from the CMS ECAL collaboration for their kind support. Furthermore, I would like to thank the organizers of the conference for the excellent organization and for the opportunity to present these results.

\section{REFERENCES}

[1] Technical Proposal. The CMS Colaboration, CERN/LHCC 94-38, LHCC/P1, 1994, The Compact Muon Solenoid (CMS).

[2] The Electromagnetic Calorimeter Project Technical Design Rep. The CMS Colaboration, CERN/LHCC 97-33, 1997.

[3] A Combination of Preliminary Electroweak Measurements and Constraints on the Standard Model, The LEP Electroweak Working Group, LEPEWWG/2003-01.

[4] E. Tournefier, "The preshower detector of CMS at LHC," Nucl. Instrum. Methods Phys. Res. A, vol. A461, pp. 355-360, 2001.

[5] E. Auffray, "Performance of ACCOS, an automatic crystal quality control system for the PWO crystals of the CMS calorimeter," Nucl. Instrum. Methods Phys. Res. A, vol. A456, pp. 325-341, 2001.

[6] J. Grahl, "Radiation hard avalanche photodiodes for CMS ECAL," Nucl. Instrum. Methods Phys. Res. A, vol. A504, pp. 44-47, 2003. 
[7] K. W. Bell, "The development of vacuum phototriodes for the CMS electromagnetic calorimeter," Nucl. Instrum. Methods Phys. Res. A, vol. A469, pp. 29-46, 2001.

[8] K. W. Bell, "The response to high magnetic fields of the vacuum phototriodes for the compact muon solenoid endcap electromagnetic calorimeter," Nucl. Instrum. Methods Phys. Res. A, vol. A504, pp. 255-257, 2003.

[9] J. Rander, "The monitoring system for the CMS-ECAL," in Proc. 8th Int. Conf. Calorimetry in High Energy Physics, Lisbon, Portugal, 1999.

[10] A. Van Lysebetten and P. Verecchia, "Performance and measurements of the light monitoring system for CMS-ECAL from 2002 test beam data," CMS NOTE, CMS RN 2004/001.
[11] L. M. Barone, "Correlation between light yield and longitudinal transmission in $\mathrm{PbWO}_{4}$ crystals and impact on the precision of the crystal intercalibration," CMS NOTE, CMS RN 2004/005.

[12] D. Futyan, "Intercalibration of the CMS electromagnetic calorimeter using jet trigger events," CMS NOTE, CMS RN 2004/007.

[13] G. Dewhirst and R. Bruneliére, "Energy resolution of the ECAL barrel super-module using MGPA electronics," CMS NOTE, CMS RN 2004/004. 